Document downloaded from:

http://hdl.handle.net/10251/100822

This paper must be cited as:

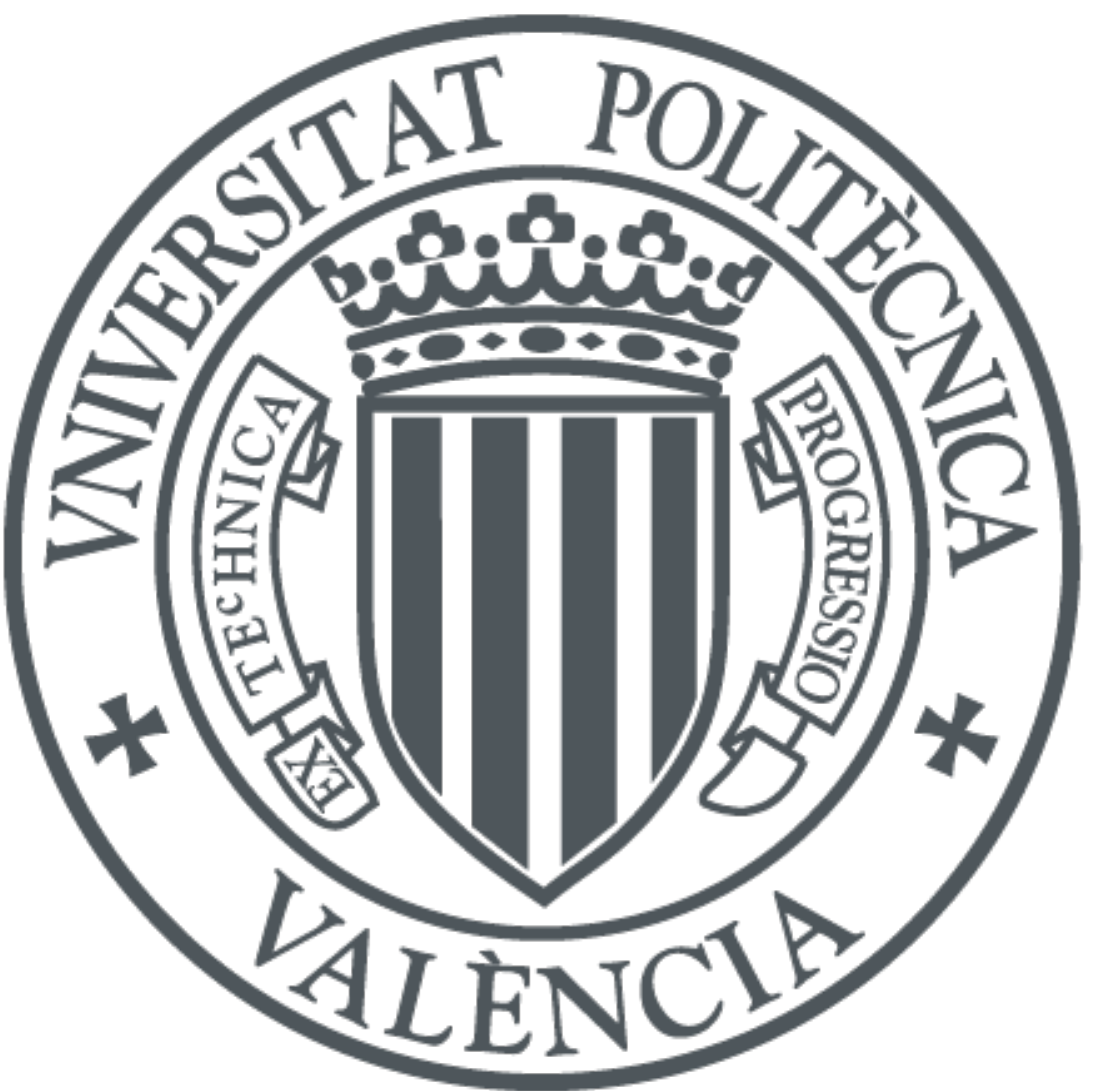

The final publication is available at

https://doi.org/10.1016/j.comnet.2017.05.018

Copyright Elsevier

Additional Information 


\title{
An Architecture and Protocol for Smart Continuous eHealth Monitoring using 5G
}

\author{
Jaime Lloret, Lorena Parra, Miran Taha, Jesus Tomás \\ ${ }^{1}$ Instituto de Investigación para la Gestión Integrada de zonas Costeras. Universidad Politécnica de \\ Valencia, Spain
}

Email: jlloret@dcom.upv.es, loparbo@doctor.upv.es, miab2@doctor.upv.es, jtomas@upv.es

\begin{abstract}
Continuous monitoring of chronic patients improves their quality of life and reduces the economic costs of the sanitary system. However, in order to ensure a good monitoring, high bandwidth and low delay are needed. The 5G technology offers higher bandwidth and lower delays and packets loss than previous technologies. This paper presents an architecture for smart eHealth monitoring of chronic patients. The architecture elements include wearable devices, to take measures from the body, and a smartphone in the patient side and a DataBase with an intelligent system which is able to send an alarm when it detects that it is happening something anomalous. The intelligent system uses machine learning in BigData taken from different hospitals and the data taken from the patient to diagnose and generate alarms. Experiment tests have been done to simulate the traffic from many users to the DataBase in order to evaluate the suitability of $5 \mathrm{G}$ in our architecture. When there is low number of users, like 100 or 200 users, we do not find big differences of round trip time between $4 \mathrm{G}$ and $5 \mathrm{G}$, but when there are more users, like 1000 users, it increases considerably reaching 4 times more in 4G. The Packet Loss is almost null in 4G until 300 users while in 5G is possible to keep it null until 700 users. Our results point out that in order to have high number of patients continuously monitored, it is necessary to use 5G network because it offers low delays and guarantees the availability of bandwidth for all users.
\end{abstract}

Index Terms - eHealth architecture, continuous monitoring, machine learning, 5G

\section{INTRODUCTION}

The number of people that need continuous monitoring because of health issues, has been increased hugely last decades. Chronical diseases, also known as non-communicable diseases, are characterized by their long duration and require systematic approaches and long -term treatments [1]. Some examples are: heart disease, cancer, chronic respiratory diseases, bone disorders, diabetes, mental disorders, vision/hearing impairment and genetic disorders among others. In 2012, in USA, the $24.3 \%$ of noninstitutionalized adults present 1 chronic condition, $13.8 \%$ present 2 and $11.7 \%$ present 3 or more [2]. Patients with chronic diseases need to spend long time periods hospitalized, being monitored. These hospitalizations suppose a reduction of the quality of life of the patients, an oversaturation of the sanitary systems and huge economic costs. According to WHO [1], the 60\% of deaths in 2005 in the world were related to chronical diseases.

Because of this, several countries started different programs to promote the health self-management. These programs improve the self-care and ensure an easy interaction between doctor and patient in order to increase the quality of life of the patient [3]. Different self-management programs were developed for different chronic diseases such as obstructive pulmonary disease [4], diabetes [5], arthritis [6], cancer [7] or heart disease [8], among others. Nowadays, the self-management consists of an essential component of the chronic care [9]. The reduction of economic cost with the current self-management programs was estimated in [10], obtaining \$364 cost saving per participant. 
United States Department of Health and Human Services pointed 4 main pillars to prevent chronical diseases. The first one is the enhanced surveillance [11]. The eHealth can increase the possibilities of patient surveillance. It is expected that eHealth becomes an essential part of the future self-management programs. With the concept of Internet of Things (IoT), which embeds computing and communication function into common objects, eHealth and other different possibilities will be benefited [12]. Nevertheless, the possibilities of wide use of sensors, the generation of Big Data, the use of artificial intelligence, the machine learning or the Cloud Computing are still generating more challenges than giving solutions.

Broadband wireless access technologies have been evolved spectacularly. 5G [13] technology, as next generation of mobile networks, will be deployed with dense small cells. It will offer significantly increased bandwidth and guarantee high Quality of Service (QoS) to the users [14]. These advantages promote 5G to provide services as IoT, cloud computing, HD 3D video streaming and interactive applications for mobile users. However, the evaluation of this technology is focused on increasing data transfer rate. Therefore, it is expected to reach 50 billion ubiquitous devices connected to the cellular network by the end of the year 2020. Furthermore, the traffic from mobile devices will account about two-thirds of the total IP traffic [15]. Therefore, the capacity of the networks must be considerably increased to satisfy these high data rates and meet excessive demands of the users without reducing the quality of service. Many applications have been designed to be adaptive and avoid to degrade the network parameters (e.g. adaptive video streaming). These applications are more flexible in order to provide integrated services in heterogeneous networks [16]. The 5G network architecture includes multiple sizes of: Femtocells, Picocells, Microcells, and Macrocells, the cover range of these cells converge from 10-20 meters, 200 meters, 2 kilometres, to 30-35 kilometres respectively and generally use diverse access technologies (UMTS, HSPA, WiMAX, LTE, and WiFi).

Wearable devices for e-health monitoring using 5G will bring several benefits to current self-management programs. The benefits for chronic patients include an enhancement of their quality of life by reducing the hospitalization time and improve their life expectancy by generating fast response alarms. The doctors will be able to review remotely their recent vital sings and physical activity in case of an alarm. Finally the reduction of hospitalization time of chronic patients will suppose a reduction of the patient's costs. The fast response to the alarms supposes a reduction of necessary surgeries due to medical complications and also a fast reply to any disease complication.

The aim of this paper is to develop an architecture for smart continuous eHealth monitoring of chronic patients using 5G. The elements of the architecture include wearable sensors and smartphones that sense the vital signs of the patient and transmit these data over the $5 \mathrm{G}$ network to a DataBase station where an intelligent engine is able to diagnose the current status of the patient. The data input of the machine learning are divided into (i) health meters such as heart rate, breathing rate or electrocardiogram from a smartphone or wearable devices, (ii) activity meters such as velocity or position from the wearable devices or smartphone, and (iii) environment parameters such as geolocation, environmental temperature, light intensity or time from the smartphone. An alert can be triggered and sent to the doctor and the intelligent engine by the patient using the alarm button. Comparing the gathered data from the patient with the BigData taken from several hospitals and the clinical history of the patient, the intelligent engine is able to diagnose the current status of the patient and generate alarms if needed. Experimental results are presented to expose the need of the 5G network in our architecture. Simulations of a specific scenario with different number of users are performed. Finally, machine learning experimental validation is performed in order to ensure its operability.

The rest of the paper is structured as follows. The related work is presented in Section II. Section III shows the proposed architecture for smart continuous eHealth monitoring over 5G network. The description of our artificial intelligence system applied to Big Data is detailed in Section IV. Section 5 presents the experimental results of $5 \mathrm{G}$ and $4 \mathrm{G}$ network simulations and the machine learning experimental validation. Finally the conclusions and future work are shown in Section VI. 


\section{RELATED WORK}

In this section a summary of the published applications of e-health using sensor devices and 5G monitoring systems are shown.

First, we review the related work of eHealth monitoring using the sensors of the smartphone or wearable sensor devices. A review of multimedia sensors included in smartphones for eHealth monitoring and ambient assisted living was done by Parra et al. in [17]. This review shows a complete picture of what type of multimedia data can be gathered and how can be used for patient monitoring. A shoe-based wearable sensor system was presented by Sazonov et al. in [18]. Their system was able to recognize the activity of the patient, e.g. sit/stand motionless, walking, ascend/descend stairs, cycling, etc. In [19], Sendra et al. proposed a smart collaborative system to monitor disabled or elderly people. Their proposal allowed recognizing the status of a person according to what was happening in his/her environment. The system was able to analyze the data of one person in conjunction with his neighbors. Lorincz et al. developed a Wireless Body Area Sensor Network (WBASN) for long-term remote monitoring of epileptic patients in [20]. Their system offered long battery lifetime and high data fidelity allowing the long-term monitoring. The WBASN was able to sense accelerometers and gyroscopes data from 8 different points of the body. A mobile phone-based pervasive fall detection system was proposed by Dai et al. in [21]. The system was implemented on an Android G1 phone. Authors compared their system with the existing ones and obtained better detection performance and power efficiency. In [22], Bravo et al. proposed a frailty monitoring by controlling daily activities. In their system, elderly people were continuously monitored using the data of the accelerometers enabled in their smartphones. By controlling their activity, the system was capable to do some recommendations for rehabilitation exercises and gave a frailty index to detect risk situations. Aminian et al. presented a monitoring system based on WBASN in [23]. Their system senses vital signals such as heart rate and the blood pressure, among other, and sends them to a base station. When abnormal conditions are detected, an alarm is generated and a sms or e-mail is sent to the caregivers. A WBASN based on a smart shirt for activity monitoring and ubiquitous health was proposed by Lee and Chung in [24]. Their system was capable to obtain an electrocardiogram and acceleration data for real time monitoring. The data was transmitted over an ad-hoc network to a basestation for remote surveillance. Gay and Leijdekkers presented a system to monitor patients with cardiac risk in [25] using WBASN and smartphone. The real-time the electrocardiogram (ECG) data was analyzed by the smartphone allowing determining if the person needs external help. The smartphone can alert the caregivers or contact with an ambulance. Moreover the system can also offer advices to the patients in order to reduce its risk (e.g. exercise more). Some systems have been proposed specifically to avoid the hospitalizations by monitoring the patient from home as in [26] and [27]. Both of them are based on WBASN.

The proposal in [26] includes a remote panic button that allows alerting the caregivers. In [28], Lloret et al. presented a smart communication architecture for ambient assisted living. The inclusion of an alert/panic button was a priority in that proposal, because although the sensors monitor the patient, some strange events cannot be detected, so it can be dangerous for the patients. In that case, the manual activation of the alert button can trigger the alarm. However, in some applications such as the one presented in [26], the alert button directly generates an alarm, but this is not efficient because sometimes the patient can activate accidentally the button generating a false alarm. To review the data in case of the alert button is pushed will help to avoid false alarms, thus saving costs, and will give better attention to the patient.

Several authors point out the amount of chances that offer 5G in conjunction with Big Data and Machine Learning to monitoring systems. In [29], Kadir et al. presented a Big Data Analytics Architecture Framework that operates under 5G. In the related literature we can find different proposals focused on 
eHealth. West presented in [30] an exhaustive summary of the benefits offered by $5 \mathrm{G}$ to eHealth. They are (i) new imaging techniques and the possibility of a second opinion thanks to high-speed transmission of X-rays or scans, (ii) tele-monitoring, which helps to obtain better diagnostics, and (iii) data mining applied to medical data, which helps to adjust the treatment among others.

We can also find some examples of proposals for eHealth operating under 5G networks. An architecture with 5G for a typical Wireless Body Area Network (WBAN) was presented by Mishra and Agrawal in [31]. Philip et al. introduced a new concept in [32], the 5G-Health as the next generation of eHealth. They discussed the possibilities of Medical Video Streaming thanks to the high speed reached in $5 \mathrm{G}$ networks.

To the best of our knowledge, there is no architecture for continuous patient monitoring over 5G that include a smart system to diagnose the status of the patient based on Big Data taken from different sanitary centres as the one that proposed in this paper.

\section{SMART CONTINUOUS EHEALTH MONITORING SYSTEM DESCRIPTION}

In this section, the developed system is presented. First, we show the design of the data gathering architecture. Then, we include the message flow and the decision algorithm for the system operation. Finally, the operation of the system when the alarm button is activated is described.

\section{A. Architecture description}

In this subsection we present the architecture of our smart continuous eHealth monitoring system over 5G. The architecture is composed by wearable devices, a smartphone and a database placed in a server. Several wireless technologies are used in the architecture. On one hand we used Bluetooth to connect sensor devices with the smartphones. On the other hand, we use $5 G$ to connect the smartphones with the Data Base (DB) server.

The system procedure is as follows. The body sensors collect information from the patient. This information is sent to the mobile device through a Bluetooth connection. The mobile device sends this information to the server through the 5G network. The DB server collects the data from the sensors. Other data are collected from hospitals, such as the medical analytics plus the decisions of the doctors. Then, the system analyses the data gathered from the sensors and the Smart Engine, using the data taken from the Big Data, decides if the data gathered are Typical Data (TD) or Abnormal Data (AD). When the system detects an abnormal situation, an alarm is generated and the server sends the last 30 minutes of data to the doctor. The doctor will verify or deny the alarm. This procedure can also be initiated by the patient through the inclusion of an alarm button. The proposed architecture is represented in Figure 1. 


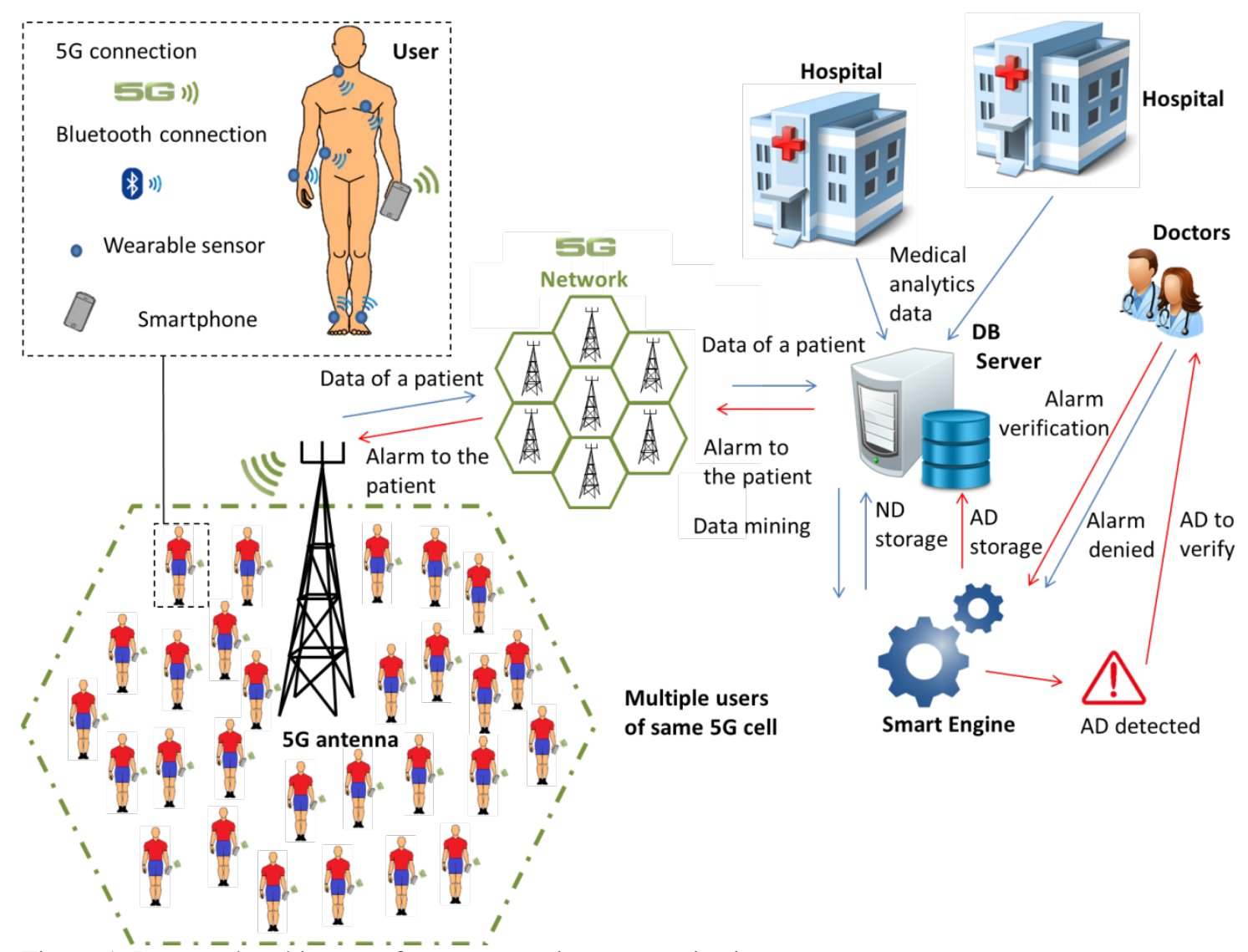

Figure 1. Proposed architecture for smart continuous monitoring

\section{B. System operation with data gathered}

This section describes the system operation when the data are sent to the Smart Engine and the different actions taken according to the result of the data analysis.

The message flow chart presented in Figure 2 shows the message flow in the case of TD detection and $\mathrm{AD}$ detection. At the beginning of the connection, the patient is registered in the $\mathrm{DB}$ and an acknowledgement (ACK) is sent from the DB to the patient to confirm the registration. From this moment the data of the wearable sensors (i.e.: heart rate, oxygen saturation, blood pressure or respiration rate among others) and of the smartphone (location, acceleration or temperature among others) are sent to the DB each second. The data from the DB are sent to the Smart Engine. A data analysis is performed by the Smart Engine using the data from the DB. The analysis offers a result. The gathered data can be TD or $\mathrm{AD}$. If the Smart Engine predicts that gathered data are TD, the message flow ends at this point. However if the Smart Engine predicts that gathered data are AD, the gathered data are sent to the doctor to verify the alarm. From this point there are two possibilities, in the first one the doctor verifies de alarm and in the second one the doctor denies the alarm. If the doctor examines the data and concludes that the alarm corresponds to a real sanitary emergency, the alarm is verified and it is considered as a Positive Alarm (PA). A verification message is sent from the doctor to the Smart Engine and the data are tagged as AD in the DB. Moreover the Smart Engine sends an alarm to the patient in order to indicate him/her the problem detected (e.g. high blood pressure detected). The message flow ends at this point in case of PA. In the second case, if the doctor concludes that the AD sent by the Smart Engine does not correspond to a real sanitary emergency, the alarm is denied and it is considered as a False Positive Alarm (FPA). A denial message is sent from the doctor to the Smart Engine and the data are tagged as TD in the DB. Then, the message flow ends at this point. 


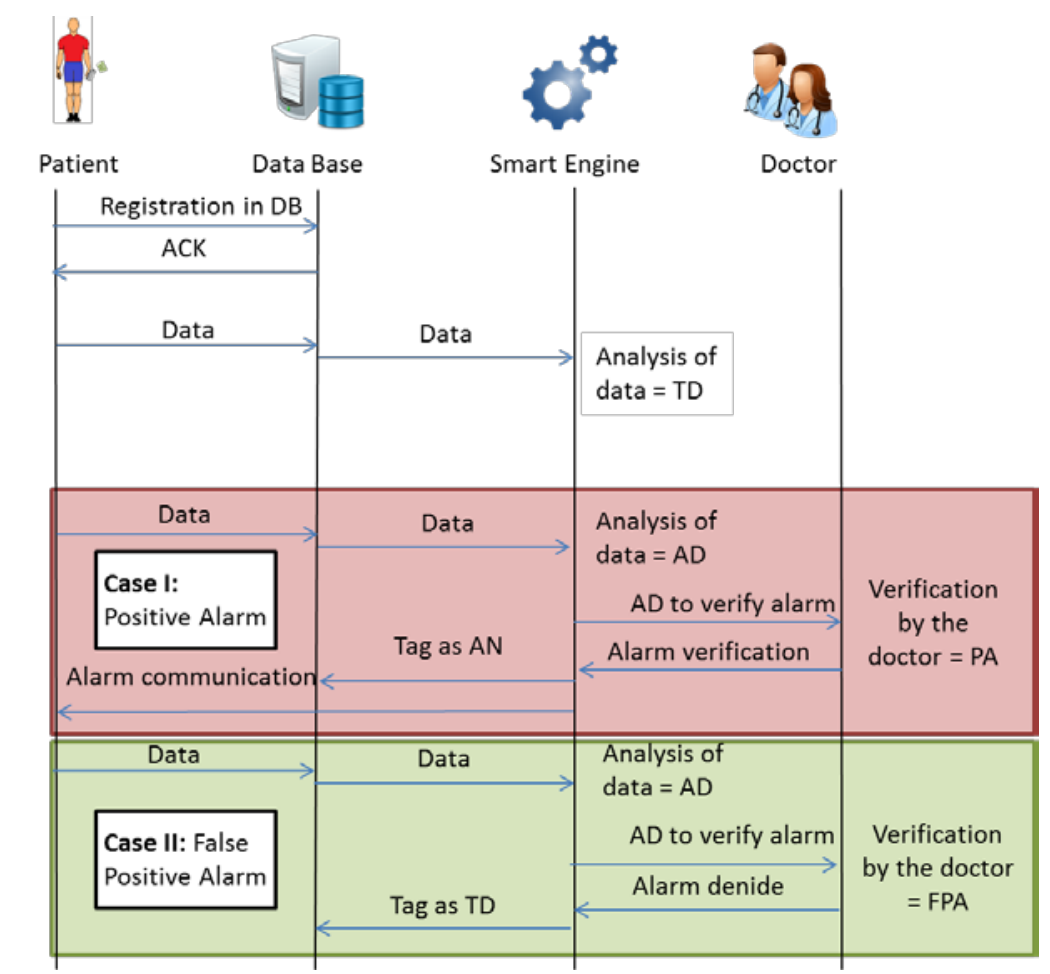

Figure 2 Message flow diagram

Figure 3 presents the decision algorithm. This algorithm is created to decide the actions to be taken after the data reception. When the gathered data are considered by the Smart Engine as TD, no further actions are taken and the data reception continues. During an episode of sanitary emergence, such as high blood pressure, the gathered data will be considered as AD by the Smart Engine, then, an alarm is created and tagged with its cause (i.e. High blood pressure detected). In order to avoid continuous messages asking for the doctor verification during a sanitary emergence event, this algorithm is able to detect if the previous data were TD or AD. If the previous data were TP it indicates the beginning of a sanitary emergence event and then the verification process will start as it is shown in Figure 2. The data are sent to the doctor in order to verify the alarm. If the alarm is verified, the data are tagged as AD. Then, the alarm is sent to the patient and the data reception continues. If the alarm is denied, the data are tagged as TD and the data reception continues. However if the previous data were already considered as AD, there are two possibilities, the current alarm can be caused by the same cause of the previous alarm or not. If both alarms have been tagged by the same cause, the new data indicate that the previous sanitary emergence event continues ongoing and it is no necessary to ask for the doctor verification, and the data reception continues without taking any action. Nevertheless if the cause of current alarm is different from the cause of the previous alarm, it indicates a new sanitary emergence event (e.g. the cause of the previous alarm is high blood pressure and the cause of the new alarm is low breathing rate) and it is necessary to ask for the doctor verification as shows Figure 2. With this algorithm it is possible to distinguish between two different sanitary emergence events and the alarm verification will be asked only when a new sanitary emergence event is detected in order to avoid repeated messages to the doctor. 


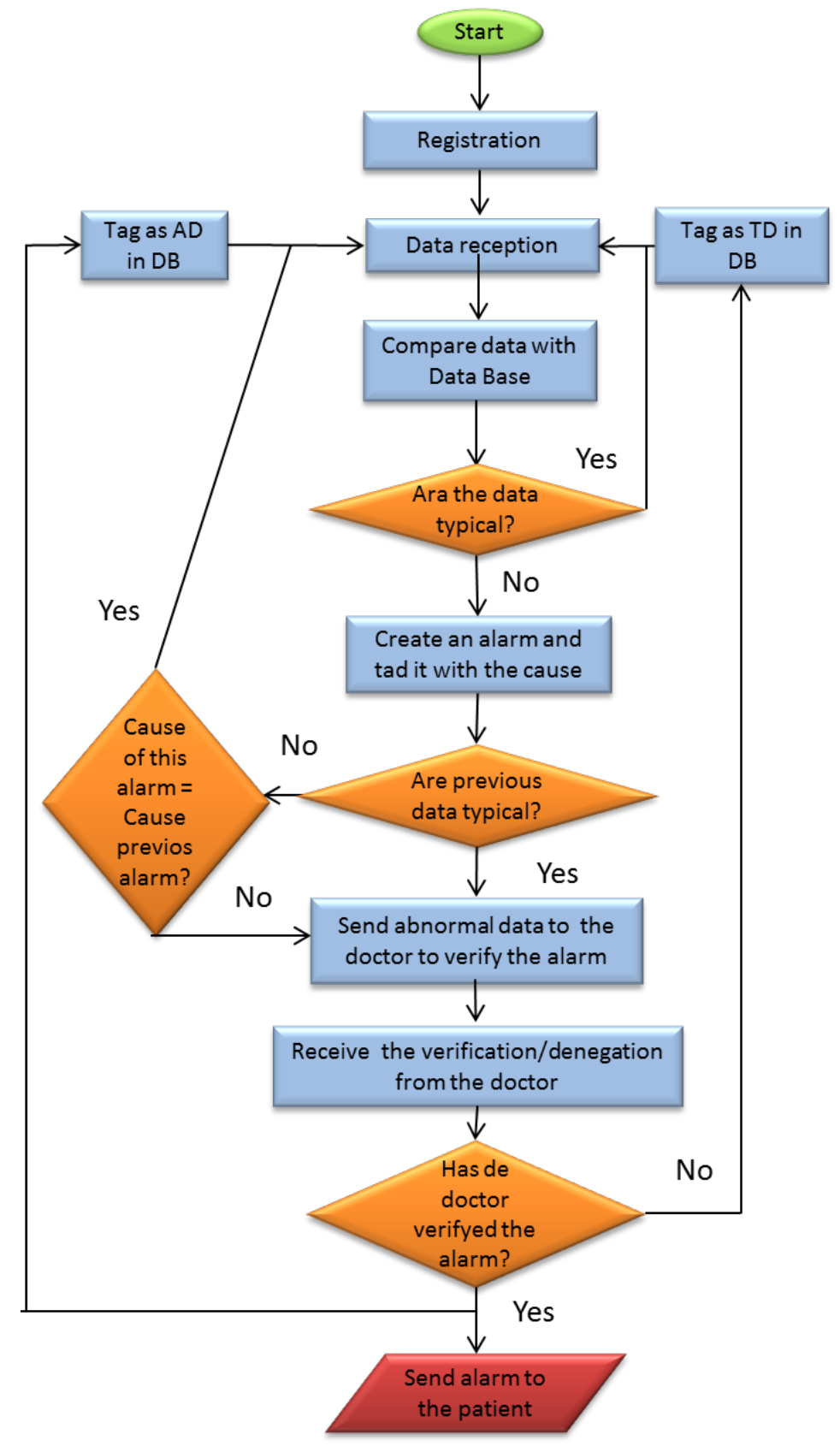

Figure 3. Decision algorithm

C. System operation with alarm button activation

In this section the process initiated by the activation of the alarm button is detailed. Current systems, that include an alarm button, use it to send an alarm to the caregivers, or to request help. However, in our system, we include the alarm button as an input of data after the doctor verification.

The process performed by the activation of the alarm button is shown in Figure 4. If during the regular data transmission process, the patient feels sick and no alarm is communicated to him, he can use the alarm button to send his last vital signs to the doctor for an evaluation. The alarm button is used by the patient to indicate an abnormal condition that was not detected by the Smart Engine. The activation of the alarm button activates the transmission of current and previous data from DB directly to the doctor. At this moment the doctor has to verify if the data pattern indicates a sanitary emerge event or not. If the doctor considers that the data indicate a sanitary emergence event, that alarm is considered as PA and proceeds to communicate the alarm to the Smart Engine and tags the data as AD into the DB. In this case, 
no alarm is sent to the patient. Using this procedure, our system is able to recognize and prevent False Negative (FN) cases. However, if the doctor considers that the received data do not correspond to a sanitary emergence event, then the alarm is considered as a FPA and no further actions are taken. Although is not usual, it is possible that the patient can unintentionally activate de alarm button, so in these cases, the caregivers will not be advised.

The decision algorithm presented in Figure 5 shows the procedure when the alarm button is activated. In this point, it is important to consider that some monitored patients can have mental diseases or other dysfunctionalities and may not be aware of the correct use of the alarm button and press it continuously.

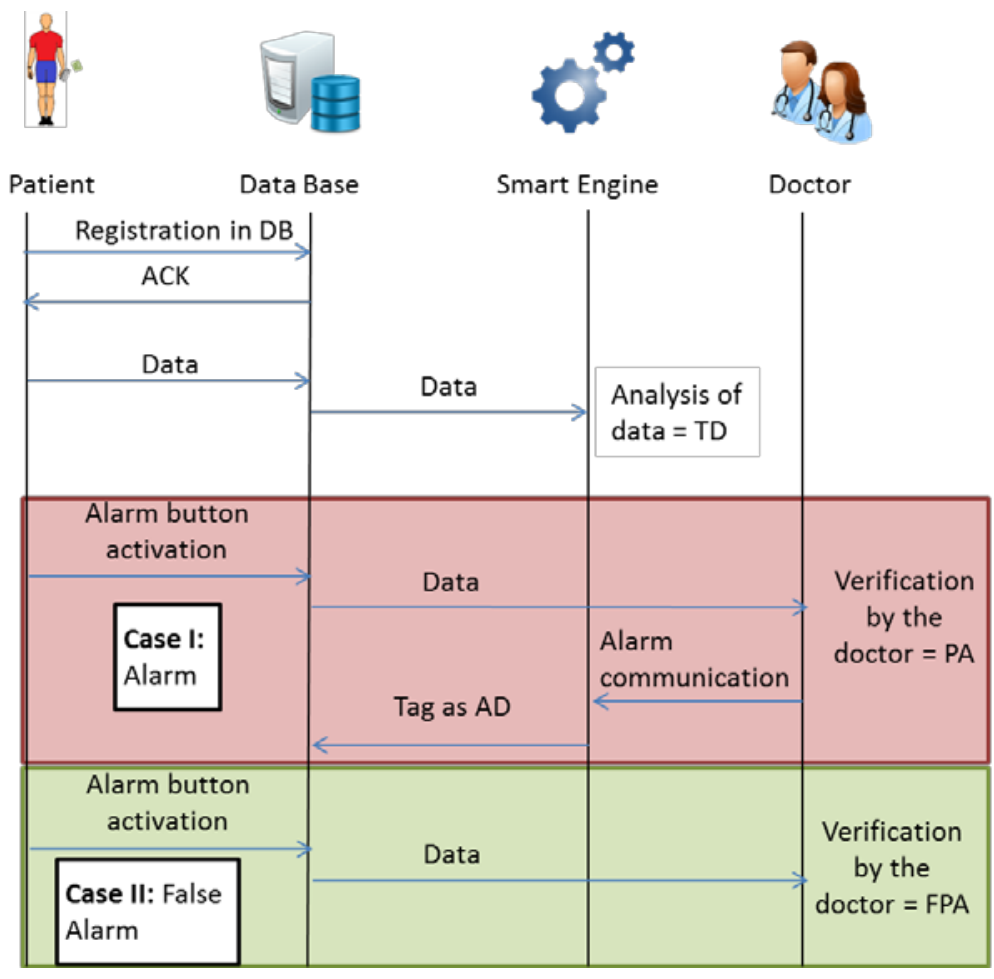

Figure 4 Message flow diagram in case of alarm button activation

To avoid continuous data exchange and continuous verification processes, the algorithm is able to discard the activation of the alarm button if it was already activated in the last 10 seconds and no further actions are taken. If the alarm was not activated in the last 10 seconds the system assumes that the patient is feeling sick and sends the data as Figure 4 shows in order to initiate the verification process. 


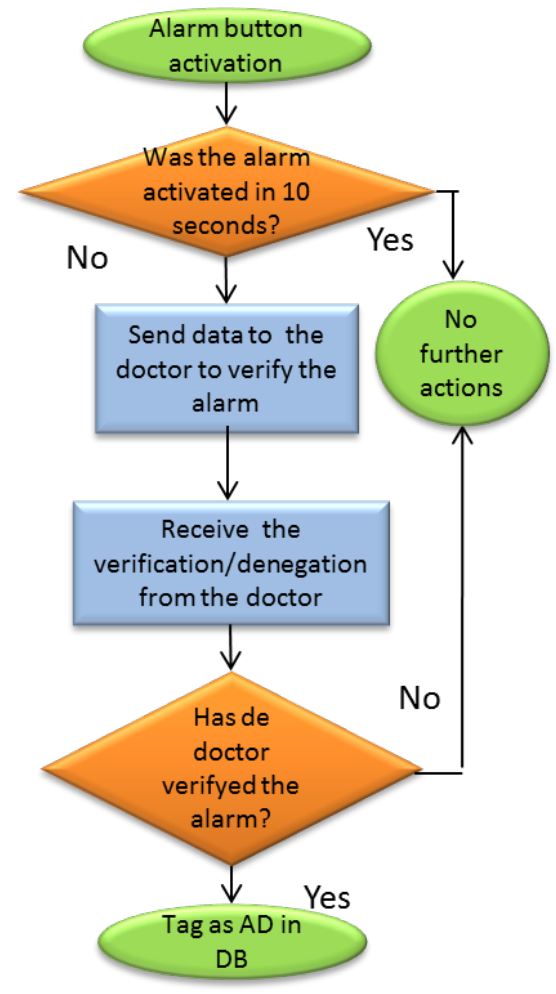

Figure 5. Decision algorithm in case of alarm button activation

\section{ARTIFICIAL INTELLIGENCE SYSTEM APPLIED TO BIG DATA}

In this section, the artificial intelligence system is presented. Given a series of inputs that monitor the patient's health parameters, our system has to be able to detect certain circumstances that require sanitary intervention.

Our system is based on inductive inference methods, that are able to anticipate the future based on past observed data. Our machine learning employs statistical techniques with the goal of enabling machines to understand the set of data. The system has been designed with a genetic algorithm, which can be applied to a wide variety of sensors and pathologies. The diagram of the developed machine learning system can be seen in Figure 6. In the following subsection, the 4 phases of our machine learning system are detailed. We select a case study focused on cardiopaty detection.

\section{A. Phase 1: Getting input data}

Input data in our system can be classified into four categories, three of them are obtained by the wearable sensors and the smartphone (health, activity and environmental parameters) and the last is the alarm button input:

- Health parameters: These sensors obtain data from human body activity directly related to the human health to be monitored. Thus, they are the main input of the system. Typically, these parameters are Electrocardiography (ECG), body temperature, sweating, heart rate, oxygen saturation, photoplethysmography, blood glucose, blood pressure or respiration rate. Our study case is focused to cardiopaty detection, thus, we used ECG and heart rate.

- Activity parameters: Health parameters are correlated to the body activity. For example, if we are running, heart rate and respiration rate increase immediately. Using an accelerometer, we can know if the patient is standing, walking, running, etc. Using a pedometer, we can measure the amount of activity. 
- Environment parameters: Other sensors can measure several parameter of the environment in which the patient is located. These parameters can also affect the health parameters. For example, we can be interested in knowing if the patient is at home or at the street, the environmental temperature, or if it is day or night. For this purpose, we incorporate a location sensor, a thermometer and a real-time clock into the system.

- Alarm button: This input is operated by the patients when they have a health problem. The medical service is warned, even if no triggers are detected. The data captured before the press can be of great help in the training phase.

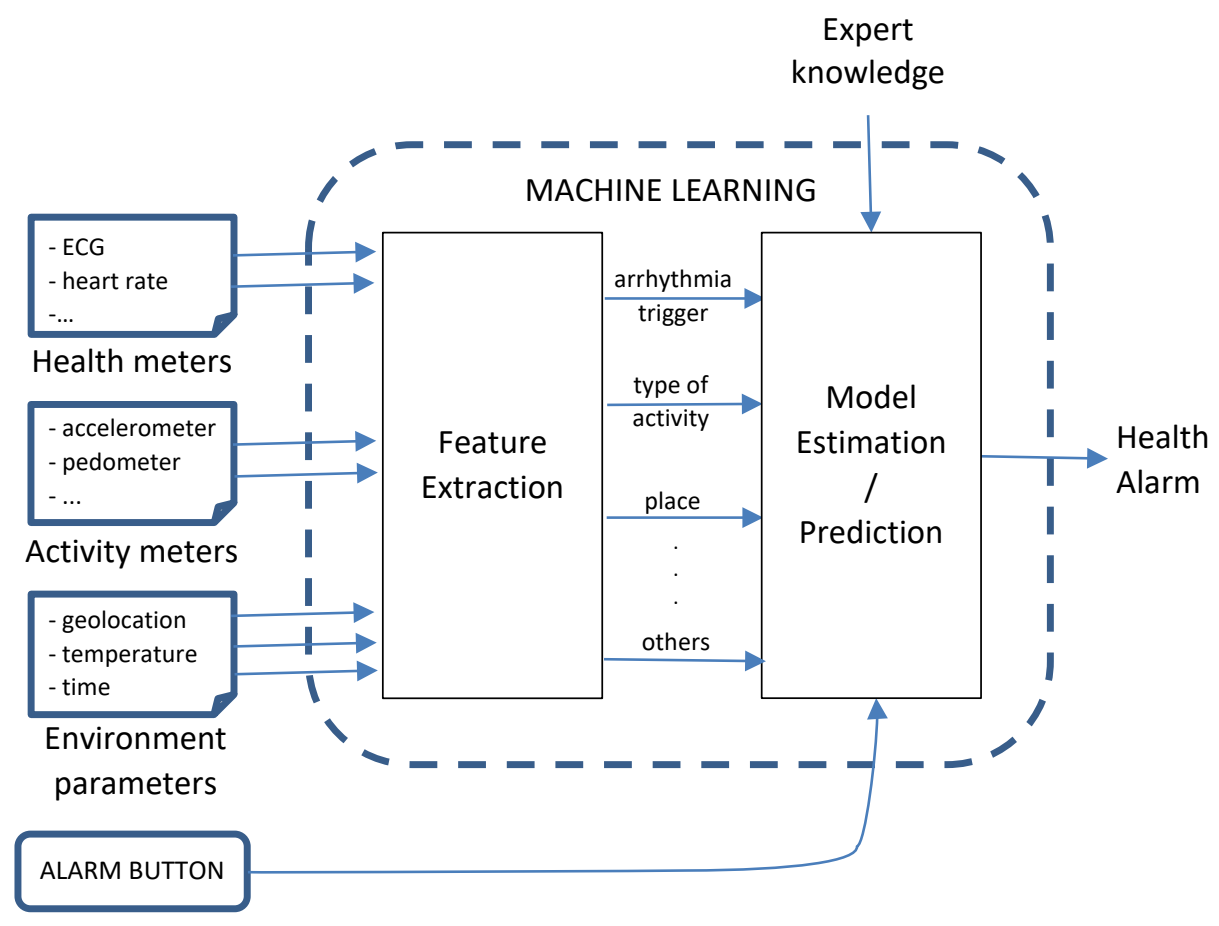

Figure 6. Machine learning diagram

\section{B. Phase 2: Feature extraction}

Body sensors can obtain very much information. Instead of working with raw data, it is more convenient to process this data in order to extract the relevant information and make the learning process easier. Features are values, derived from initial data, intended to be informative and non-redundant, facilitating the learning process and the generation of the models.

In our system, several input parameters can be passed to the next phase without pre-processing. For example, we can use heart rate, pedometer and temperature. However, other parameters must be transformed into a more understandable form. Below we describe the obtained features:

- Arrhythmia: There are many works that describe how to detect arrhythmia pathologies from the ECG. In our system, we use an arrhythmia classificatory similar that described in [33]. For training the models we use the MIT-BIH database [34].

- Type of activity: Using an accelerometer we can detect the activity of the patient. Possible values for this feature are: standing, walking, running, cycling, driving or sleeping. Other sensors, like heart rate, can help to detect this feature [33].

- Place: The location of the patient can be relevant for the system. A location sensor gives us some position coordinates. However, a more concrete place can be more informative for the system. In a domestic implementation we can differentiate three places: home, outdoor, indoor. In a hospital implementation we can differentiate: patient room, WC and rest room of the hospital. 


\section{Phase 3: Learning}

As an initial learning phase, we propose a deductive learning method, using a system based on rules. The expert's knowledge is directly included in the system through a series of decision rules. In order to make this rules we mainly consider the arrhythmia notification and, in some cases, the hearth rate and the type of activity.

Even when the previous method works adequately, the process to set the umbral where the alarm must be triggered is a difficult process. It is necessary to consider many examples to be able to decide the best value.

Furthermore, an expert can only consider a limited set of inputs. The time of day or the patient's location can be invaluable in order to establish a rule. Because of those reasons, we consider that an inductive method can obtain better results than the one based on rules.

For this purpose, we use a multilayer perceptron (MLP). It is a feedforward artificial neural network. For training the perceptron, we use the data of different alarms, where it is annotated manually if it is a false positive or not. Data taken from situations with no alarm are also used. In a no supervised learning process, we use additional inputs, like pedometer, place, temperature and time.

\section{Phase 4: Prediction}

When the system is running, both the rule based and the inductive methods run in parallel. We may use one or other information depending on the degree of training of the system. We can configure the system to trigger the alarm when any of the learned cases happens. Even if no alarms are triggered, all patient data is stored for improve the training and for monitoring purposes.

\section{EXPERIMENTAL RESULTS}

In this section the obtained results are shown. First, a description of the simulated scenario is described. Then, the results of the simulations are presented and the suitability of 5G network is discussed. Finally, the verification of our proposed intelligent system is demonstrated.

\section{A. Scenario}

This subsection describes the experiments in 4G/LTE and 5G wireless network technologies. We have designed a topology that includes the wireless network, a high-performance HTTP, reverse proxy, and IMAP/POP3 proxy (NGINX) server and mobile users. The NGINX server is configured to receive HTTP requests. Specifically, we generate two types of traffic; TCP -based network traffic and ICMP -based network traffic. Moreover, tools such as Iperf, ping, curl and wireshark are used to generate traffic with different packet sizes and to activate the measurement of the Quality of Service (QoS) parameters. It reports the bandwidth, delay, packet loss and jitter.

Therefore, in our experiments, we select several sizes of packets. Moreover we took measurements for different number of packets: 10 packets, 100 packets and 1000 packets. On the other hand, we generate arbitrary TCP traffic to demonstrate the available throughput of each studied technology.

In order to evaluate the performance we send data packets from the mobile users to the database server through the $4 \mathrm{G}$ or $5 \mathrm{G}$ base stations. They have different available bandwidth, delay and packet loss ratio.

B. 5G and 4G simulation results

The simulation of the traffic between the client and the server using 4G and 5G technologies is presented in this subsection. Different tests are done in order to evaluate the performance of both networks when the 
number of users increases. The evaluated parameters are round trip time, bandwidth and packet lost.

In the first experiment, the 4G network parameters includes; 4Mbps of available bandwidth, a delay of 20 milliseconds and $0.001 \%$ packet loss rate. The network parameters of the $5 \mathrm{G}$ are $1000 \mathrm{Mbps}$ with a delay that ranges from 1 to 5 milliseconds with rate of $0.0001 \%$ packet loss. Mobile users upload data to the central server through different wireless characteristics. Moreover, we generate sensor data traffic from many mobile users, which range between 100 and 1000 users. These mobile users separately send data traffic, with different packet sizes, to the server. Figure 7 shows that $5 \mathrm{G}$ connection wastes less round trip time than 4G connections. On the other hand, the average delay in 5G for high-connected mobile users is less than 10ms. Otherwise, in 4G the delay has higher value. When there are 1000 users, for 10 packets we obtained 30ms, for 100 packets there was more than $40 \mathrm{~ms}$ and for 1000 packets about $80 \mathrm{~ms}$.

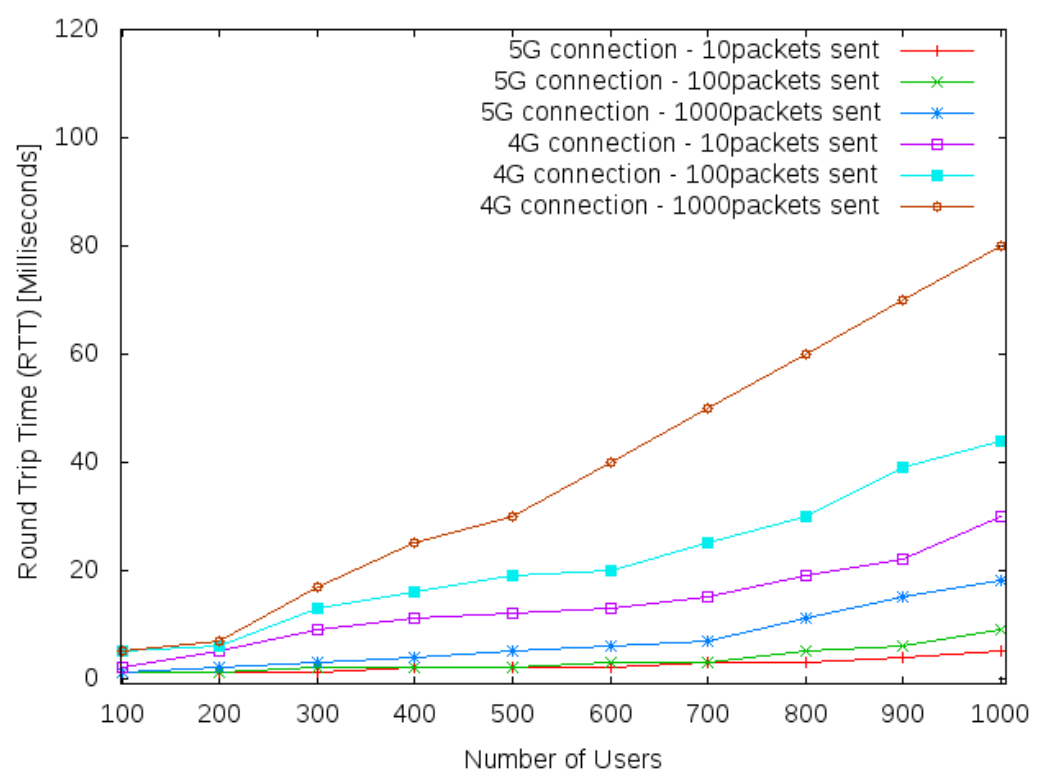

Figure 7. Round trip time of packets in $4 \mathrm{G}$ and $5 \mathrm{G}$ base stations.

In the second experiment, we set the $5 \mathrm{G}$ network parameters as: 1000Mbps of bandwidth, delay variation between 1 and 5 milliseconds and packet loss rate of $0.1 \%$. In $4 \mathrm{G}$, there is a bandwidth of 20Mbps, a delay variation between 2 and 40 milliseconds and a packet loss rate of $0.1 \%$. The results of this test are shown in Figure 8. It shows number of lost packets per sample when data is sent using these technologies. On the one hand, we observe that in $4 \mathrm{G}$ all cases have a packet loss and these rates are increased when the number of mobile users increases, because the traffic increases. These cases present higher number of packet loss than 5G. However, in 5G technology there are more packets lost in the cases when there are more users sending data to the central server as shows the yellow line, range from 700 to 1000 users. Therefore, packet loss in 5G is smaller than the range of packet loss in 4G. 


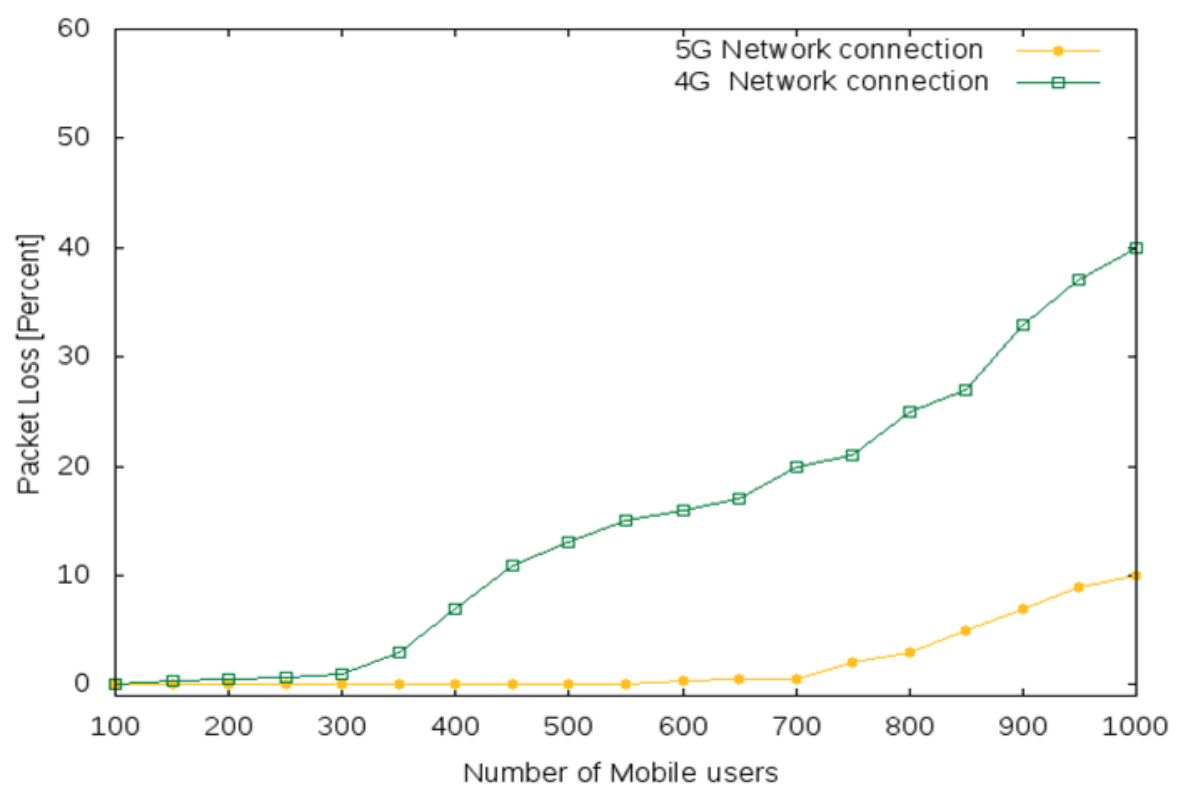

Figure 8. Packet loss rate in 4G and 5G technologies.

Figure 9 shows the number of packets retransmitted in the TCP connection experiment. We observe what happens when a mobile user sends data in both $4 \mathrm{G}$ and $5 \mathrm{G}$ technologies. In 5G, the variation of the throughput ranges from $1000 \mathrm{Mbps}$ to $500 \mathrm{Mbps}$, the delay varies between 1 and 5 milliseconds and the packets lost rate is $0.01 \%$. In the $4 \mathrm{G}$ technology, the throughput ranges from $20 \mathrm{Mbps}$ to $40 \mathrm{Mbps}$, the delay varies between 2 and 40 milliseconds and the packet loss rate is $0.01 \%$. The blue line of Figure 5 shows the number of packets retransmitted by the TCP protocol. In 4G, the number of packets retransmitted is higher than the retransmission in $5 \mathrm{G}$ as depicted in the graph, the red line at the $35^{\text {th }}$ second has only one packet lost.

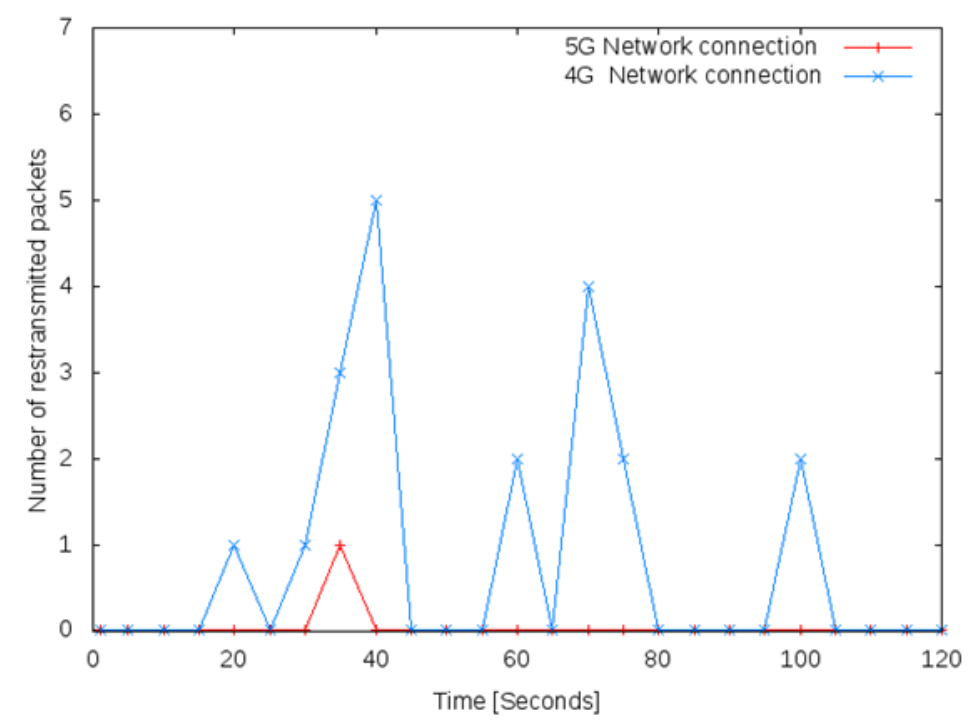

Figure 9. Packet retransmission in 4G and 5G.

In the last experiment, we generate arbitrary traffic in both, 5G and 4G technologies. They have set the following parameters. $5 \mathrm{G}$ has $1000 \mathrm{Mbps}$, delay from 1 to 5 milliseconds, with $0.0001 \%$ loss rate and $4 \mathrm{G}$ has $200 \mathrm{Mbps}$, delay from 2 to 40 milliseconds, with $0.001 \%$ loss rate. Figure 10 shows the network throughput of both technologies for different amount of mobile users. The green line indicates the throughput of 5G. It shows that the throughput has been decreased with the increase of traffic nodes but, the range of throughput has not reached to threshold. On the other hand, the blue line arrives the threshold point when more than 300 users are connected to the base station sending data in the $4 \mathrm{G}$ network. 


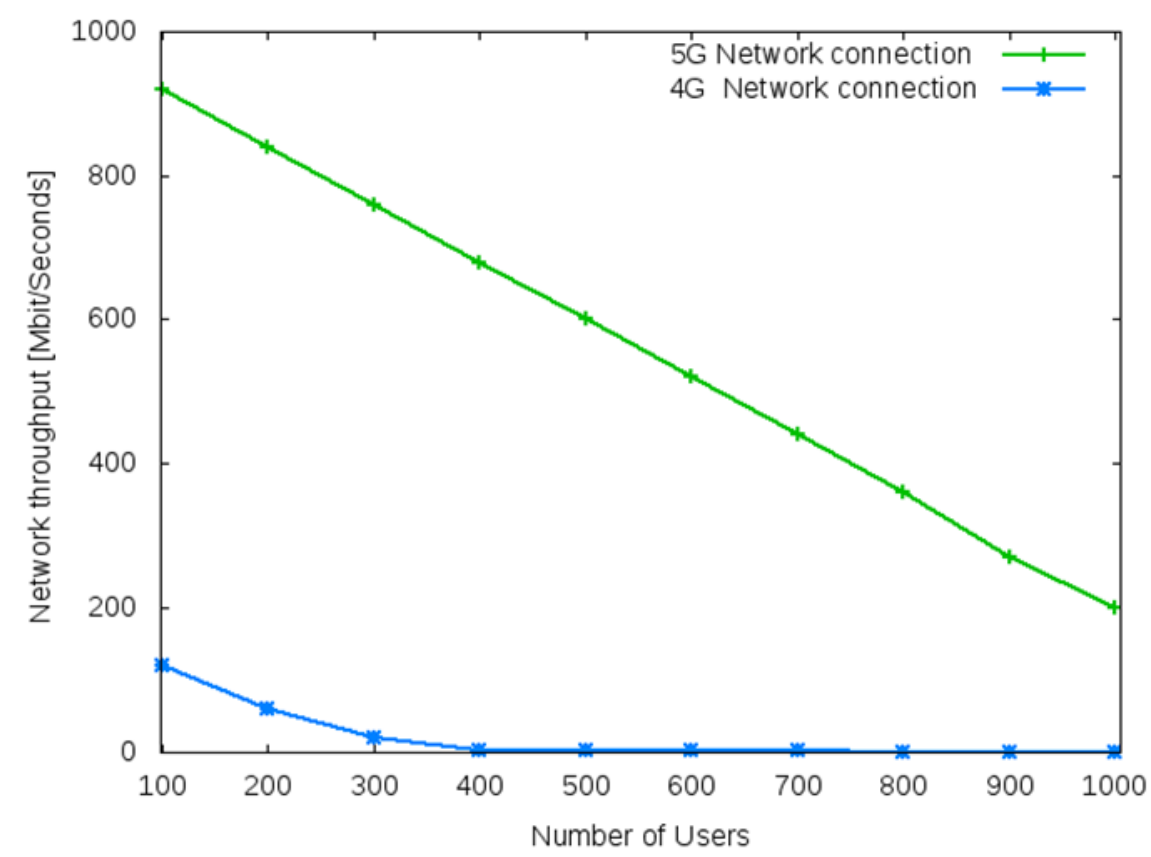

Figure 10. 4G and 5G throughput.

\section{Machine learning experimental validation}

In this section the validation of our intelligent system is shown. To validate the system, we implemented a prototype focused in the detection of cardiopathies. Cardiopathies are one of the most important chronic diseases to monitor because its serious effects on the health and the possibility of death after a heart attack. Moreover, it is simple to monitor the cardiac signs of a patient.

In order to perform the experimental validation of our systems, we have carried out a series of experiments. We have used as training corpus. The data was captured from 6 patients that suffered cardiological pathologies. The acquisition was performed from 7 to 12 days, depending on the patient. The data has been manually marked by medical experts. In total, 132 relevant events (arrhythmias, taquicardies, etc.) were obtained. Not all of those have to mean an alarm for the medical services. We estimated that only between 5 to $10 \%$ of those events correspond with an urgency that should be notified.

In order to train the system, we used the data from one patient for testing and the other 5 patients for training. This procedure is repeated for the 6 patients. Figure 11 shows the results of the neural network (NN) and its comparison with the rule based (RB) system. Using the RB system we obtain a correct prediction in $80 \%$ of the cases. Using the $\mathrm{NN}$ the results improve up to $85 \%$. This improvement can make a better fitting of the thresholds. In order to improve the prediction, we introduced new inputs. Adding the feature activity, the correct prediction, we have a successful prediction rate of $87 \%$. Adding the feature place, temperature or time, no improvement was observed. 


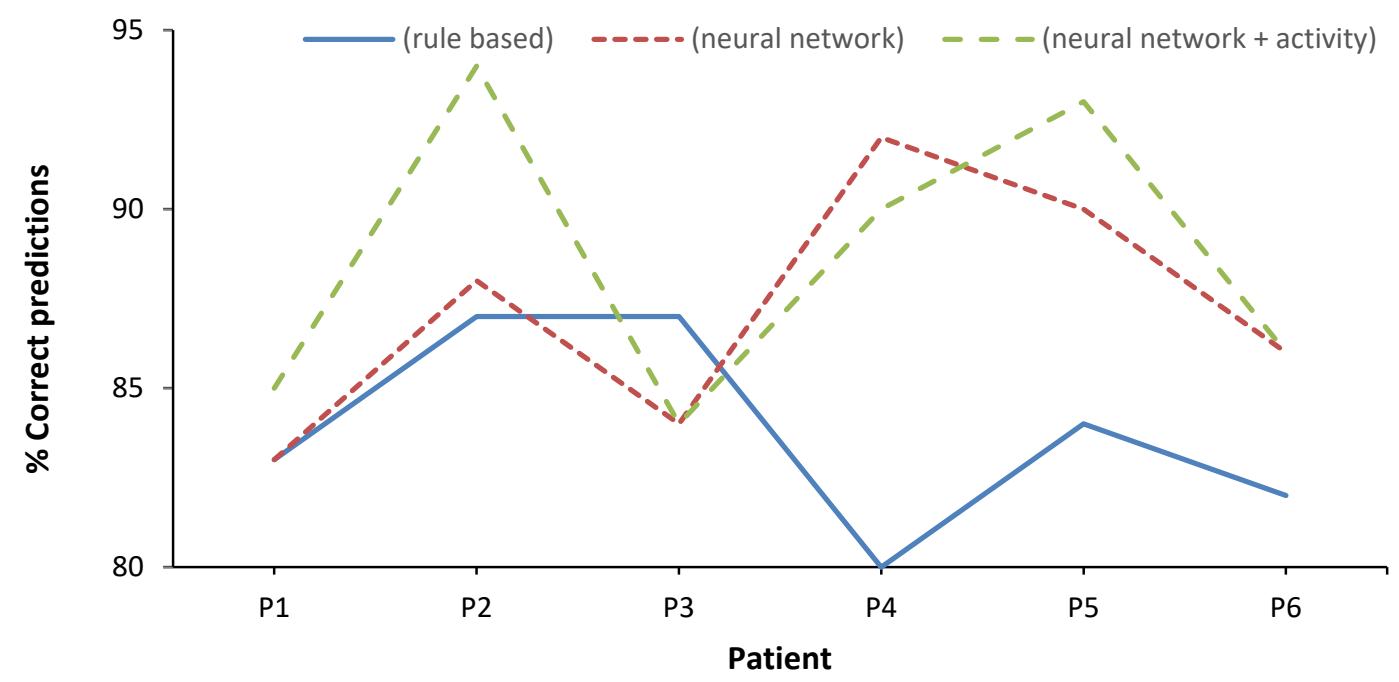

Figure 7. Results of the neural network and its comparison with the rule-based system.

\section{ConcLusions}

The need of continuous patient monitoring is a well-known reality. However if we pretend to have a smart monitoring with high speed data exchange and fast response, 5G networks are needed. In this paper, we develop architecture and protocol for smart continuous eHealth monitoring. We create an intelligent system, which is able to diagnose patients and generate alarms based on artificial intelligence analysis over Big Data. We simulated 4G and 5G technologies in order to demonstrate the need of 5G technology in our architecture. 4G technology does not support huge traffic load especially when the number of users simultaneously send request data through the base stations. Moreover, high delay and many packet retransmissions produce high latency of packets, which affects the users because they will get late response of the requests. We also verified the developed intelligent system using a case study based on cardiac diseases.

The main advantages of our system are:

- The system can be triggered by an alarm button or by the detection of AD by the Smart Engine.

- Two message flow diagrams and two decision algorithm have been designed for the proper operation of our system in case of data reception and in case of alarm button activation.

- The Smart Engine learns from PA and FPA by asking the doctor about the verification of the gathered data.

- The use of an alarm button by the patient, followed by the positive doctor verification will help our system to learn from FN.

- The first algorithm created distinguishes when a new sanitary emergence event starts for its first time (it is not one previously repeated some seconds ago) from one that has been caused by other reason.

- The second algorithm is able to detect the misuse of the alarm button and discard the alarm

Our future work is focused on training the intelligent system with the data of each patient separately. We also want to include different types of alarms depending on the severity of the alarm. Moreover, a summary of different wearable devices and a study of their suitability for specific diseases monitoring will be done. Furthermore, we would like to let our system send recommendations to the patients when an alarm is sent (for example, low blood pressure is detected, the system sends a recommendation like "increase the liquids ingestion and avoid the abrupt changes of position". 


\section{ACKNOWLEDGMENT}

This work has been partially supported by the “Ministerio de Educación, Cultura y Deporte”, through the “Ayudas para contratos predoctorales de Formación del Profesorado Universitario FPU (Convocatoria 2014)”. Grant number FPU14/02953.

\section{REFERENCES}

[1] WORLD HEALTH ORGANIZATION, et al. Preventing chronic diseases: a vital investment: WHO global report. 2005.

[2] B. W. Ward, "Multiple chronic conditions among US adults: a 2012 update”. Preventing chronic disease, vol. 11, 2014

[3] J.E. Jordan, R.H. Osborne, "Chronic disease self-management education programs: challenges ahead”, Medical Journal of Australia, vol. 186, no 2, p. 84. , 2007

[4] T. EFFING, Tanja, E.E.M. Monninkhof, P.P.D.L.P.M. van der Valk, G.G.A. Zielhuis, E.H. Walters, J.J van der Palen, M. Zwerink "Self-management education for patients with chronic obstructive pulmonary disease”, Cochrane Database Syst Rev, vol. 4, 2007

[5] J.A. Gregg, G.M. Callaghan, S.C. Hayes, J.L. Glenn-Lawson, "Improving diabetes self-management through acceptance, mindfulness, and values: a randomized controlled trial”, Journal of consulting and clinical psychology, vol. 75, no 2, p. 336, 2007

[6] K.R. Lorig, P.L. Ritter, D.D. Laurent, K. Plant, "The internet-based arthritis self-management program: A one-year randomized trial for patients with arthritis or fibromyalgia", Arthritis Care \& Research, vol. 59, no 7, p. 1009-1017, 2008

[7] R. McCorkle, E. Ercolano, M. Lazenby, D. Schulman-Green, L.S. Schilling, K. Lorig, E.H. Wagner, "Self-management: Enabling and empowering patients living with cancer as a chronic illness", CA: A Cancer Journal for Clinicians, vol. 61, no 1, p. 50-62, 2011

[8] L.P. Dale, R. Whittaker, Y. Jiang, R. Stewart, A. Rolleston, R. Maddison, “Text message and internet support for coronary heart disease self-management: results from the Text4Heart randomized controlled trial”,. Journal of medical Internet research, vol. 17, no 10, 2015

[9] M.W. Huygens, J. Vermeulen, I.C. Swinkels, R.D. Friele, O.C. van Schayck, L.P. de Witte, "Expectations and needs of patients with a chronic disease toward self-management and eHealth for selfmanagement purposes”, BMC Health Services Research, vol. 16, no 1, p. 232, 2016

[10] S. Ahn, R. Basu, M.L. Smith, L., Jiang, L. Lorig, N. Whitelaw, M.G. Ory, “The impact of chronic disease self-management programs: healthcare savings through a community-based intervention”, $B M C$ Public Health, vol. 13, no 1, p. 1, 2013

[11] U.E. Bauer, P.A. Briss, R. A., Goodman, B.A., Bowman, "Prevention of chronic disease in the 21st century: elimination of the leading preventable causes of premature death and disability in the USA", The Lancet, vol. 384, no 9937, p. 45-52, 2014

[12] F. Xia, L.T. Yang, L. Wang, A. Vinel, “Internet of things”, International Journal of Communication Systems, vol. 25, no 9, p. 1101, 2012

[13] M. Ruffini, "Multi-Dimensional Network Convergence in Future 5G Networks", Journal of Lightwave Technology i, 2016 
[14] Z. Xi; C. Wenchi; Z. Hailin, "Heterogeneous statistical QoS provisioning over 5G mobile wireless networks." IEEE Network, vol. 28, no 6, p.46-53, 2014

[15] R. Gartner Inc., Predicts 2016: The Internet of Things [online] available: http://www.gartner.com/ document/2952822. [Accessed: Dec. 2016]

[16] L. García, J. Lloret, C. Turro, M. Taha, "QoE assesment of MPEG-DASH in polimedia eLearning System”, 5th International Conference on Computing, Communications and Informatics (ICACCI), Jaipur, India, September, 2016, p. 1117-1123.

[17] L. Parra, S. Sendra, J.M. Jiménez, J. Lloret, "Multimedia sensors embedded in smartphones for ambient assisted living and e-health” Multimedia Tools and Applications, p. 1-27, 2015

[18]E.S. Sazonov, G. Fulk, J. Hill, Y. Schutz, R. Browning, "Monitoring of posture allocations and activities by a shoe-based wearable sensor", IEEE Transactions on Biomedical Engineering, vol. 58, no 4, p. 983-990, 2011

[19] S. Sendra, E. Granell, J. Lloret, J.J. Rodrigues, "Smart collaborative mobile system for taking care of disabled and elderly people”, Mobile Networks and Applications, vol. 19, no 3, p. 287-302, 2014

[20] K. Lorincz, B.R. Chen, G.W. Challen, A.R. Chowdhury, S. Patel, P. Bonato, M. Welsh, "Mercury: a wearable sensor network platform for high-fidelity motion analysis”, SenSys, p. 183-196, 2009

[21] J. Dai, X. Bai, Z. Yang, Z. Shen, D. Xuan, “Mobile phone-based pervasive fall detection”, Personal and ubiquitous computing, vol. 14, no 7, p. 633-643, 2010

[22] J. Bravo, F. Navarro, J. Fontecha, R. Hervas, "A mobile proposal for frailty monitoring by rehabilitation and physical daily activity", In Proccedings of the 1st IEEE International Conference on Consumer Electronics - Berlin (ICCE-Berlin 2011), Berlin, Germany, 6 - 8 September, 2011, p. 176-180.

[23] M. Aminian, and H. Naji, "A hospital healthcare monitoring system using wireless sensor networks", J. Health Med. Inform, vol. 4, p. 121, 2013

[24] Y.D. Lee and W.Y. Chung, "Wireless sensor network based wearable smart shirt for ubiquitous health and activity monitoring”, Sensors and Actuators B: Chemical, vol. 140, no 2, p. 390-395, 2009

[25] V. Gay and P. Leijdekkers, "A health monitoring system using smart phones and wearable sensors", International Journal of ARM, vol. 8, no 2, p. 29-35, 2007

[26] J.V. Lee, Y.D. Chuah, K.T. Chieng, "Smart elderly home monitoring system with an android phone", Int. J. Smart Home, vol. 7, no 3, p. 17-32, 2013

[27] S. González-Valenzuela, M. Chen, V. Leung, "Mobility support for health monitoring at home using wearable sensors", IEEE Transactions on Information Technology in Biomedicine, 2011, vol. 15, no 4, p. 539-549, 2011

[28] J. Lloret, A. Canovas, S. Sendra, L. Parra, “A smart communication architecture for ambient assisted living”, IEEE Communications Magazine, vol. 53, no 1, p. 26-33, 2015

[29] E.A. Kadir, S.M. Shamsuddin, T.A. Rahman, A.S Ismail, "Big Data Network Architecture and Monitoring Use Wireless 5G Technology”, Int. J. Advance Soft Compu. Appl, vol. 7, no 1, p. 1-14, 2015

[30] D.M. WEST, "How 5G technology enables the health internet of things", Brookings Center for Technology Innovation, vol. 3, p. 1-20, 2016

[31] A. Mishra and D.P. Agrawal, "Continuous health condition monitoring by $24 \times 7$ sensing and transmission of physiological data over 5-G cellular channels", In Proceedings of the International 
Conference on Computing, Networking and Communications (ICNC 2016), Kauai, Hawaii, USA, 15-18 February, 2016, p. 584-590.

[32] N.Y. PHILIP and I.U.REHMAN, “ Towards 5G Health for Medical Video Streaming over Small Cells”, In Proceedings of the XIV Mediterranean Conference on Medical and Biological Engineering and Computing, Paphos, Cyprus, 31 March - 2 April, 2016, p. 1087-1092

[33] H. Banaee, M.U. Ahmed, A. Loutfi, "Data mining for wearable sensors in health monitoring systems: a review of recent trends and challenges”, Sensors, vol. 13, no 12, p. 17472-17500, 2013

[34]G.B. Moody and R.G. Mark, “The impact of the MIT-BIH arrhythmia database”, IEEE Engineering in Medicine and Biology Magazine, vol. 20, no 3, p. 45-50, 2001. 